\title{
Melanocytic Nevus of the Tarsal Conjunctiva
}

\author{
Bülent Yazıc1 ${ }^{1}$, Ayşe Dolar Bilge ${ }^{1}$, Ayşe Yağc1 ${ }^{2}$, Faisal Naqadan ${ }^{1}$, Filiz Altıntepe ${ }^{3}$ \\ ${ }^{1}$ Department of Ophthalmology, Uludağ University School of Medicine, Bursa, Turkey \\ ${ }^{2}$ Department of Ophthalmology, Ege University School of Medicine, İzmir, Turkey \\ ${ }^{3}$ Bursa Pathology Center, Bursa, Turkey
}

Background: Melanocytic nevus is a rare occurrence in the tarsal conjunctiva and only 7 well-described cases have been reported previously in the English literature.

Case Report: The medical records of 4 patients with tarsal conjunctival melanocytic nevus were reviewed, together with the relevant literature. All patients (3 women and 1 man; age range: 17 - 40 years) had been referred with a suspicion of melanoma. There was one tarsal nevus in the lower eyelid in 3 patients and 2 nevi in the upper eyelid in 1 patient. All lesions were darkly pigmented with irregular borders and were associated with a history of a recent growth in size. An intralesional cyst was present in 1 nevus only. After surgical excision, no recurrence or complication occurred during the follow-up period (range: $7-48$ months).

Conclusion: Tarsal melanocytic nevus has been described in detail in 11 cases, including these 4 cases, in the English literature. The lesion arose from the lower eyelid in all cases except one. Tarsal melanocytic nevi may frequently display clinical features suggesting melanoma, such as advanced patient age, recent growth, dark and irregular pigmentation, nodularity, hypervascularity, and the absence of an intralesional cyst. After total excision, nevus recurrence or malignant trans-formation has not been reported.

Keywords: Conjunctiva, conjunctival nevus, melanocytic nevus, tarsal plate
Melanocytic nevi occur frequently in the epibulbar, caruncular, and plical conjunctiva. They are very rare in the tarsal conjunctiva, and the clinical characteristics of this subgroup of patients are not well delineated $(1,2)$. In this report, 4 cases of tarsal conjunctival melanocytic nevus are presented, together with the relevant literature. Written informed consent was obtained from all patients for the use of clinical data and images.

\section{CASE PRESENTATION}

\section{Case 1}

A 17 year-old, healthy, light-skinned woman was referred, with a suspected melanoma in the right lower eyelid. The patient reported that the black spot that had been present from her childhood had grown in the past 2 years. A black, nodular le- sion, $7 \times 6 \mathrm{~mm}$ in diameter on the tarsal surface and lid margin was noted in the central part of the lower eyelid (Figure 1a). Increased peri- and intralesional vascularization and cystic formations were noted in the inferior margin of the lesion. With the patient under local anesthesia, a full-thickness eyelid excision was performed, and the defect was closed primarily with the addition of lateral canthotomy and cantholysis. Histologically, the lesion was diagnosed as subepithelial compound nevus (Figure 1e). No nuclear atypia or mitosis was observed. Nevus recurrence, malignant transformation, or complication did not develop during the 35 month follow-up.

\section{Case 2}

A 21 year-old woman noted a dark lesion in her right lower eyelid 3 months previously. She was referred by an ophthalmologist with a possible diagnosis of melanoma. The patient was light skinned and healthy. On examination, a 

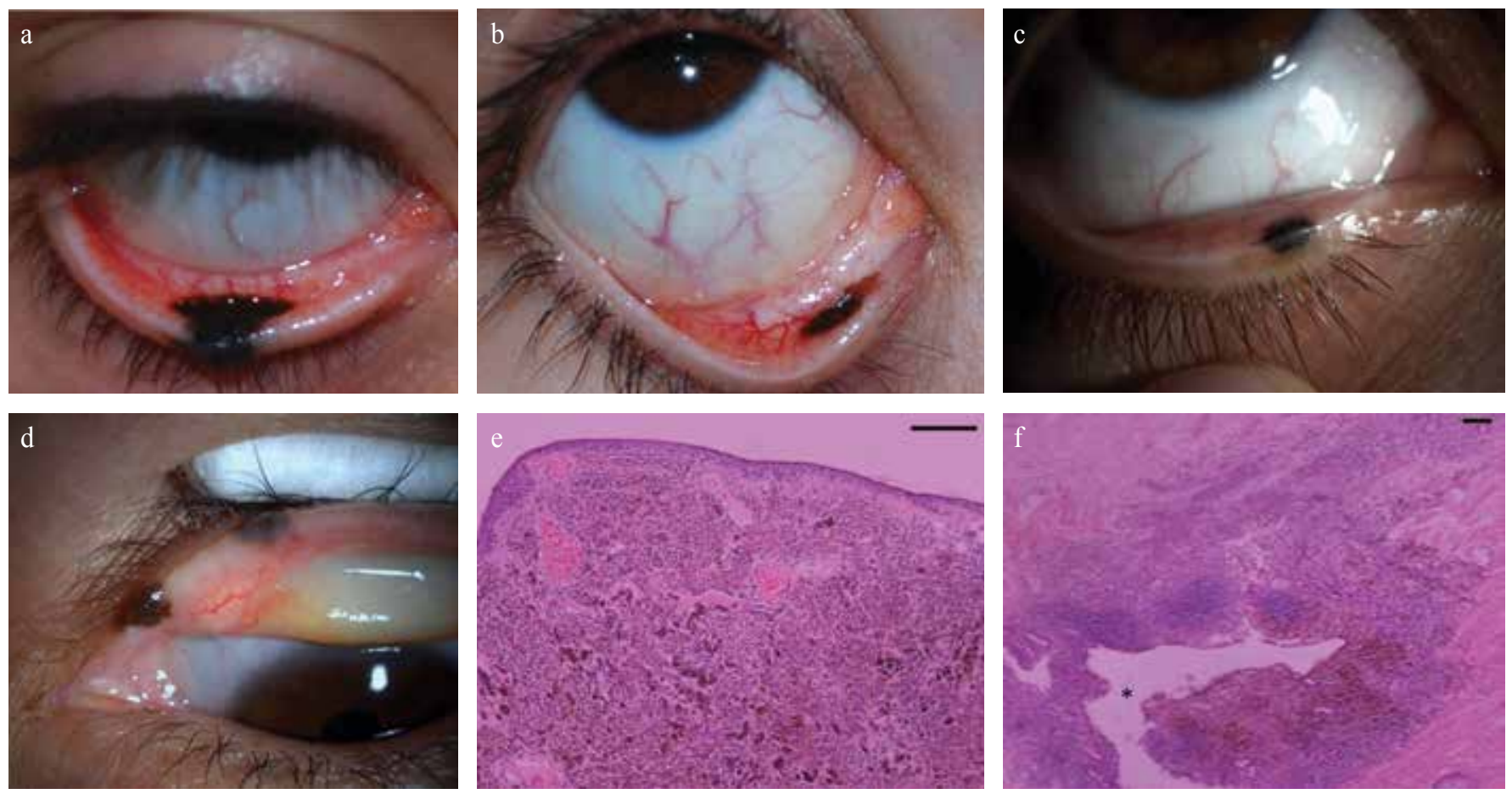

FIG. 1. a-f. Nodular melanocytic nevus in the tarsal conjunctiva and lid margin in the lower eyelid in a 17 year-old patient (a), the clinical appearance of the tarsal melanocytic nevus in case 2 (b), case 3; a tarsal melanocytic nevus in the right lower eyelid (c), case 4; two tarsal melanocytic nevi in the left upper eyelid (d), case 1; histologic section showing subepithelial compound nevus composed of small epitheloid-type nevus cell groups, dark, pigmented melanophages, and dentritic melanocytes (hematoxylin-eosin, scale bar indicates $100 \mu \mathrm{m})(\mathrm{e})$, case 2; inflamed compound nevus consisting of nevus cells, abundant infiltration of lymphocyte, plasmocyte, eosinophil leukocytes, and lymphoid follicular structures (hematoxylin-eosin, scale bar indicates $100 \mu \mathrm{m}$ ) (f), asterisk indicates the lacrimal canalicular lumen.

black-brown, slightly elevated lesion with irregular borders, $7 \times 3$ in diameter, located behind the lower punctum, was noted (Figure 1b). The lesion had feeding and intrinsic vessels, but no intralesional cysts. The lesion was totally excised under local anesthesia. The posterior lamellar defect was repaired with a tarsal graft taken from the contralateral upper eyelid. Histopathology reported an inflamed compound nevus (Figure 1f). The postoperative follow-up period of 40 months was without complications.

\section{Case 3}

A 40 year-old woman presented with a black-brown lesion measuring $2 \times 3 \mathrm{~mm}$ in size, involving the tarsal conjunctiva and posterior lid margin on the medial side of the right lower eyelid (Figure 1c). She had first noted this lesion 3 years previously, and was concerned about the risk of a malignancy, which had been mentioned by another ophthalmologist. The lesion had irregular pigmentation, intrinsic vessels, and no intralesional cysts. The lesion was excised with full-thickness eyelid resection and described histologically as dysplastic compound nevus. The follow-up period of 7 months was uneventful.

\section{Case 4}

A 34 year-old male patient was referred with a suspicion of melanoma in the left upper eyelid. Upon examination, two, slightly elevated, dark-brown lesions were noted in the medial eyelid margin, extending to the tarsal conjunctiva (Figure 1d). Both lesions had irregular pigmentation and borders, and no intrinsic vessels or cysts. The patient underwent excisional biopsy with full-thickness eyelid resection and defect reconstruction using semicircular flap. Histological diagnosis was junctional nevus for both lesions. No recurrence and complication developed during the 48 month follow-up.

\section{DISCUSSION}

Melanocytic nevi of the tarsal conjunctiva are rare. In 2 studies including a total of 761 conjunctival nevi, 3 nevi involved the eyelid tarsus $(0.4 \%)(3,4)$. In addition, Kim et al. (5) found a total of 10 cases in the English literature until 2010 and reported 4 new cases. Of these cases, only 7 had detailed clinical and histologic information $(1,2,5)$. In this report, a total of 11 cases including the 4 presented herein are reviewed.

Epibulbar conjunctival nevi are frequently diagnosed between 10 and 19 years of age (4). However, tarsal nevi are noticed at a later age. Among the 11 cases described in the literature, the median patient age was 49 years at the time of diagnosis (range: 
TABLE 1. Several characteristics of 4 patients with tarsal conjunctival nevi

\begin{tabular}{lccccc}
\hline & Age (yr) - Gender & Location & Intralesional Cysts & Histological Description & Follow-up Time (mo) \\
\hline Case 1 & $17, \mathrm{~F}$ & Right, LL & Yes & Subepithelial compound nevus & 35 \\
Case 2 & $21, \mathrm{~F}$ & Right, LL & No & Inflamed compound nevus & 40 \\
Case 3 & 40, F & Right, LL & No & Dysplastic compound nevus & 7 \\
Case 4 & $34, \mathrm{M}$ & Left, UL & No & Junctional nevus & 48 \\
\hline
\end{tabular}

F: female; M: male; LL: lower lid; UL: upper lid

5-70 years; mean age, 42 years). Six patients were men and 5 were women. Eight patients were Caucasian in origin, and the others were Asian, Hispanic, and African. The most frequent symptom was the growth of a pigmented spot, which was reported in 8 patients. The duration of symptoms ranged from 1 month to 10 years in 7 patients and was unclear in the others. Interestingly, in all but one of the patients, the lesion occurred in the lower eyelid. It was on the right side in 7 patients (64\%). The nevus also involved the lid margin in 6 patients $(54.5 \%)$ and it was only on the tarsal surface in the others. All except 2 nevi were slightly elevated from the tarsus. The lesions were black-brown or gray in color in 9 patients. The pigmentation was described as "peppered" and "partial" in 2 patients (1). Intralesional cysts were noted in $3(33 \%)$ of 9 patients, and feeding and/or intrinsic vessels in 5 patients (56\%); no information about these features was available in 2 patients (1) (Table 1). In all cases including ours, the referral diagnosis was melanoma or suspicion of melanoma. We doubted this diagnosis on the basis of clinical findings and choose to excise the lesions with a narrow normal tissue margin, as performed in cases of epibulbar nevi. We did not use cryotherapy or topical chemotherapy in any case. Extensions of excision and method of repair have not been stated in the previous reports. Postoperative follow-up data were available for 8 patients and no nevus recurrence or malignancy developed in any case between 7 and 48 months.

It appears that conjunctival nevi may display melanoma-like clinical features more frequently in the tarsus than in the other areas. Intralesional cysts, which are a benign sign, were relatively rare in tarsal nevi compared with epibulbar nevi (33\% vs $65 \%)$ (4). However, the presence of feeding and intralesional vessels is a feature raising the concern of melanoma and may be relatively frequent in tarsal nevi (56\% vs 33\%) (4). Besides, the "negative" features such as advanced patient age, dark pigmentation, nodularity, border irregularity, and recent growth may be more prevalent in tarsal nevi (1). Nevertheless, only a few reports specifically evaluated the tarsal melanocytic nevi, and more patients are needed to confirm these comparisons.

On the basis of the current data, for differential diagnosis of tarsal melanocytic lesions, the most reliable approach would be excisional biopsy $(1,4)$. It should be considered that melanomas arising from the palpebral conjunctiva are associated with a 2.2-times higher mortality rate compared with epibulbar melanomas $(6,7)$.

\section{Ethics Committee Approval: N/A.}

Informed Consent: Written informed consent was obtained from patients who participated in this study.

Peer-review: Externally peer-reviewed.

Author contributions: Concept - B.Y.; Design - B.Y., A.D.B., A.Y.; Supervision - B.Y., A.Y., A.D.B., F.A.; Resource - B.Y., A.Y., F.A.; Materials - B.Y., A.D.B., A.Y., F.N., F.A.; Data Collection and/or Processing - B.Y., A.D.B., Y.A., F.N., F.A.; Analysis and/or Interpretation B.Y., A.D.B., F.A., F.N.; Literature Search - B.Y., A.D.B., F.N.; Writing - B.Y., A.D.B., F.N.; Critical Reviews - B.Y., A.D.B., Y.A., F.N., F.A.

Conflict of Interest: No conflict of interest was declared by the authors.

Financial Disclosure: The authors declared that this study has received no financial support.

\section{REFERENCES}

1. Buckman G, Jakobiec FA, Folberg R, McNally LM. Melanocytic nevi of the palpebral conjunctiva: an extremely rare location usually signifying melanoma. Ophthalmology 1988;95:1053-7. [Crossref]

2. Obata H, Kubota M, Kawai T, Tsuru T. Nevocellular nevus of the palpebral conjunctiva. Acta Ophthalmol Scand 2004;82:6323. [Crossref]

3. Gerner N, Nørregaard JC, Jensen OA, Prause JU. Conjunctival naevi in Denmark 1960-1980. A 21-year follow-up study. Acta Ophthalmol Scand 1996;74:334-7. [Crossref]

4. Shields CL, Fasiuddin AF, Mashayekhi A, Shields JA. Conjunctival nevi: clinical features and natural course in 410 consecutive patients. Arch Ophthalmol 2004;122:167-75. [Crossref]

5. Kim HJ, McCormick SA, Nath S, Moskowitz B, Milman T. Melanocytic nevi of the tarsal conjunctiva: Clinicopathologic case series with review of literature. Ophthal Plast Reconstr Surg 2010;26:438-42. [Crossref]

6. Paridaens AD, Minassian DC, McCartney AC, Hungerford JL. Prognostic factors in primary malignant melanoma of the conjunctiva: clinicopathological study of 256 cases. $\mathrm{Br} \mathrm{J}$ Ophthalmol 1994;78:252-9. [Crossref]

7. Shields CL, Markowitz JS, Belinsky I, Schwartzstein H, George NS, Lally SE, et al. Conjunctival melanoma: outcomes based on tumor origin in 382 consecutive cases. Ophthalmology 2011;118:389-95. [Crossref] 